\title{
Differential transplantability of human endothelial cells in colorectal cancer and renal cell carcinoma primary xenografts
}

\author{
Laura Sanz ${ }^{1}$, Ángel M Cuesta ${ }^{1}$, Clara Salas ${ }^{2}$, César Corbacho $^{2}$, Carmen Bellas $^{2}$ and Luís Álvarez-Vallina ${ }^{1}$
}

In vivo models of human tumor vasculature are essential for the study of tumor angiogenesis and validation of therapeutic targets. To date, however, few standardized animal models of human tumor angiogenesis have been characterized. It was recently shown that human renal cell and prostate carcinoma primary xenografts, established from biopsy specimens, contained vessels lined mainly by human endothelial cells 1 month after implantation in immunodeficient mice. We selected colorectal cancer (CRC) as a primary xenograft model and studied the response of the vascular compartment to the new microenvironment during the same lapse of time. Immunohistochemical analysis of the origin of endothelial cells demonstrated that, in contrast to the mentioned study, human endothelial cells were rapidly substituted by their murine counterparts (nearly $50 \%$ by day 10 after implantation). Apoptotic human endothelial cells could not be detected 10 days after implantation, suggesting that apoptosis is not the mechanism underlying their replacement. Interestingly, host endothelial cells were found to colocalize with human laminin, suggesting a colonization of human vascular basement membranes after human endothelial cell disappearance. To rule out that the differences observed between the fate of human vasculature in the CRC model and those previously reported were because of methodological aspects, we established renal cell carcinoma (RCC) primary xenografts using the same protocol. In clear contrast with CRC xenografts, vasculature within RCC xenografts was mostly of human origin 35 days after implantation. These results support the notion of angiogenic heterogeneity in malignant neoplasms. Elucidation of the molecular mechanisms that determine persistence or disappearance of human endothelial cells in different tumor contexts can help to shed light on the intimate regulation of the angiogenic process.

Laboratory Investigation (2009) 89, 91-97; doi:10.1038/labinvest.2008.108; published online 10 November 2008

KEYWORDS: animal model; endothelium; primary human xenograft; tumor angiogenesis; tumor microenvironment; vascular remodeling

It is widely accepted that tumors cannot grow or metastasize without the recruitment of new blood vessels, and the angiogenic switch is a key early event in tumor progression. ${ }^{1,2}$ The idea that tumors could be starved to death by inhibiting their neovascularization was first proposed more than 30 years ago by Folkman, and the first antiangiogenic agents have been recently approved. ${ }^{3}$

Angiogenesis is a multistep process implying complex molecular and cellular interactions that cannot be recapitulated in vitro. ${ }^{4,5}$ Because of this, animal models are essential for the study of the angiogenic process and validation of therapeutic targets. Unfortunately, there are few standardized in vivo models of human tumor angiogenesis. ${ }^{6}$ Xenografts of human tumor cell lines into immunodeficient mice are valuable tools in cancer research and have provided clinically relevant experimental models for the development and testing of new therapeutic agents. However, the vascular component of tumors in these experimental models is provided by the murine host, and therefore is not adequate to study the effect of antiangiogenic strategies in human endothelium. ${ }^{7}$ Conversely, long-lasting functional human blood vessels can be engineered in nude mice after implantation of human primary endothelial cells (ECs), ${ }^{8-13}$ but they lack the influence of the tumor component. Coimplantation of tumor cells along with human ECs provides a best-suited model of tumor vasculature, ${ }^{14}$ but it is not an accurate surrogate of the complex tumor microenvironment.

Alternatively, primary tumor xenografts imply the transplantation of tumor tissue fragments containing the original vasculature. ${ }^{15}$ Generally, tumor cells from patient biopsy

\footnotetext{
${ }^{1}$ Molecular Immunology Unit, Hospital Universitario Puerta de Hierro, Madrid, Spain and ${ }^{2}$ Pathology Department, Hospital Universitario Puerta de Hierro, Madrid, Spain Correspondence: Dr L Sanz, MD, PhD, Molecular Immunology Unit, Hospital Universitario Puerta de Hierro, San Martín de Porres 4, Madrid 28035, Spain.

E-mail: Isanz.hpth@salud.madrid.org

Received 29 July 2008; revised 29 August 2008; accepted 29 August 2008
} 
specimens xenografted into mice preserve their original histological and biological characteristics, and different groups have tried to correlate engraftment and growth of human implants in conditioned or nonconditioned mice with stage, grade and proliferation index of primary tumors, with different success rates. ${ }^{16,17}$ However, the fate of ECs embedded in these implants has been scarcely studied. In a seminal work, Gray et $\mathrm{al}^{18}$ reported that the majority of vessels in human renal cell carcinoma (RCC) and prostate adenocarcinoma primary xenografts are still lined with human ECs by day 30 after implantation. What is more, vessel density and number of vessels with proliferating ECs increased during this lapse of time in prostate adenocarcinoma xenografts.

Colorectal cancer (CRC) is one of the most common malignancies in the western world. As none of the existing mouse models mimics all the characteristics of human CRC, ${ }^{19}$ we explored the engraftment of human specimens in nude mice as an alternative with special attention paid to the vascular compartment. The evolution of blood vessels inside the tumors was followed for a month. In contrast to other primary xenograft models, we assessed a rapid substitution of human ECs lining vessels by their murine counterparts that did not take place in RCC xenografts implanted following the same protocol.

\section{MATERIALS AND METHODS \\ Mice}

Five-week-old female athymic nude mice (Hsd: athymic Nude/Nude; Harlan Iberica, Barcelona, Spain) were used for xenograft implantation. All animals were maintained in a sterile environment. Cages, bedding, food and water were autoclaved. Anesthesia was induced by intraperitoneal injection of a combination of ketamine (Ketolar ${ }^{\circledR}$, Pfizer, New York, NY, USA) and diazepam (Valium ${ }^{\circledR}$, Roche, Nutley, NJ, USA). All experimentation involving laboratory animals was approved by the Institutional Animal Care and Use Committee and performed in accordance with national legislation.

\section{Tissue Collection and Implantation}

Fresh surgical specimens were obtained immediately after surgery, inspected by a pathologist to select viable tissue and kept in DMEM (Invitrogen, Carlsbad, CA, USA) at $4^{\circ} \mathrm{C}$. Patients did not receive neoadjuvant treatment before surgery that could compromise tissue integrity. Measures were taken to maintain sterility of tissue from the time of resection until implantation, being always this lapse of less than $2 \mathrm{~h} / \mathrm{im}$ mediate in most cases. An initial tissue specimen was cut from each sample, placed in $10 \%$ formalin for fixation and paraffin embedded. The remainder of each specimen was cut in $2-4 \mathrm{~mm}^{3}$ pieces, embedded in Matrigel (BD Biosciences, Erembodegem, Belgium) and implanted subcutaneously in the flanks of anesthetized mice, 3-4 pieces per mouse. For implantation, a 14-gauge trocar device was used without the need of a previous incision.

\section{Histology and Immunohistochemical Study}

At different time-points after implantation mice were killed, and the xenografts were removed, formalin fixed and paraffin embedded. A histological section from each initial tissue specimen and each xenograft was stained with hematoxylin and eosin (H\&E) according to standard protocols. A mouse monoclonal antibody $(\mathrm{mAb})$, reacting with human cytokeratins 1-6, 8, 10, 14-16, 19 (clone AE1/AE3, Dako, Glostrup, Denmark) was used to confirm the presence of tumor cells. Human ECs were visualized with a mouse $\mathrm{mAb}$ antihuman CD34, clone QBEnd10 (Dako) and a mouse mAb antihuman CD31, clone JC70A (Dako), using the Mouse on Mouse (M.O.M. ${ }^{\mathrm{TM}}$ ) peroxidase kit (Vector Laboratories, Burlingame, CA, USA). Standard avidin-biotin complex peroxidase immunohistochemistry staining for mouse CD31 (clone MEC 13.3, BD Biosciences) and mouse CD34 (clone MEC14.7, Immunotools, Friesoythe, Germany) was performed on serial sections with Vectastain ${ }^{\circledR} \mathrm{ABC}$ kit for rat IgG (Vector Laboratories). As a chromogenic substrate, DAB (Vector Laboratories) was used, followed by hematoxylin counterstaining. Double immunostaining of human EC apoptosis was performed as previously described ${ }^{20}$ with the mouse mAb antihuman CD34, clone QBEnd10 and rabbit anti-active caspase-3 polyclonal antibody (BD Biosciences) using the EnVision ${ }^{\text {TM }}$ G/2 Doublestain system, rabbit/mouse $(\mathrm{DAB}+/$ permanent red) from Dako. For human laminin colocalizaton with mouse CD34, we used the mouse $\mathrm{mAb}$ clone LAM-89 (Sigma, St Louis, MO, USA) after antigen retrieval with pepsin (Sigma) $2 \mathrm{mg} / \mathrm{ml}$ in $0.01 \mathrm{M} \mathrm{HCl}, 30 \mathrm{~min}$ at $37^{\circ} \mathrm{C}$. Negative controls were performed in parallel, with omission of the primary antibody incubation step.

\section{Analysis of Blood Vessel Density}

Sections stained with antihuman or antimouse CD34 mAbs were first scanned at low magnification $(\times 50)$ to identify vascular 'hot spots'. Areas of greatest vessel density were then examined under higher magnification $(\times 200)$ and counted. $^{21}$ Five high-power fields (HPF) per section were examined. Results were expressed as the mean number of microvessels \pm s.d. per HPF. Brown-staining areas identified by the DAB staining, whether single ECs or clusters of ECs, regardless of the absence or presence of a lumen were counted as individual microvessels.

\section{RESULTS \\ Establishment of Human Colorectal Cancer Primary Xenografts}

Colorectal cancer xenografts were established from histologically intact patient specimens by subcutaneous implantation into athymic nude mice. Xenografts were harvested after 10,20 or 30 days in the host animal and were evaluated for viability at the time of harvest. Tumors were considered as engrafted when tumor tissue could be detected macroscopically at the time of resection and histologic analysis of tumor morphology and tumor marker expression 
(cytokeratins) revealed the presence of viable tumor cells (data not shown). Engrafted xenografts were pink-to-red in color, indicating anastomosis to the host vasculature and perfusion. The proportion of surgical specimens that yielded at least one viable xenograft was $62.5 \%$, with per fragment take rates of nearly $50 \%(n=38)$.

The histology of the xenografts was consistent with the initial tissue specimens (Figure 1a-c), although atrophic glands surrounding extracellular mucin pools were observed in specimens recovered 30 days after implantation. Xenografts at this time also contained areas of hemorrhage and necrosis that were not observed in their corresponding initial tissue specimens. Xenograft volume increased with time, consistently with proliferation of CRC cells and/or formation of large lakes of extracellular mucin. Although implanted specimens had a maximum volume of $4 \mathrm{~mm}^{3}$, xenografts generally reached at day 30 postimplantation more than $250 \mathrm{~mm}^{3}$.

\section{Vasculature in the Xenografts and Comparison of Vessel Density}

The central aim of this study was to assess the fate of human vasculature during xenograft establishment compared with the corresponding initial tissue specimens. For this purpose, xenografts were removed at different time points after implantation, stained with $\mathrm{H} \& \mathrm{E}$ and evaluated histologically. In initial tissue specimens as well in xenografts, the vasculature was found both adjacent to glands and distributed through the stroma. By day 10, blood vessels in the xenografts already contained erythrocytes, indicating successful anastomosis with the host vasculature (Figure 1e). Blood vessels filled with erythrocytes were consistently detected in sections from days 20 and 30 (Figure if and data not shown). To study the evolution of vessel density, average number of vessels per field was quantified in sections of xenografts from days 10, 20 and 30 postimplantation as well as in initial tissue specimens. No significant differences in vessel density were noted between initial specimens and xenografts. The consistent observation of areas of necrosis in the xenografts harvested at day 30 postimplantation indicated that reperfusion of initial specimens was sufficient to support establishment of the xenografts but inadequate to ensure viability of the entire implant when it reached a critical size.

\section{Species-Specific Vascular Labeling}

CD31 and CD34 are classical markers of ECs. Serial sections of initial CRC tissue were stained for human CD31 (Supplementary Figure 1a) and CD34 (Supplementary Figure 1b) to establish which marker detected the largest number of vessels in the xenografts with similar results. The species specificities of these mouse mAbs antihuman CD31 and CD34 were checked by IHC analysis of human and mouse control tis-

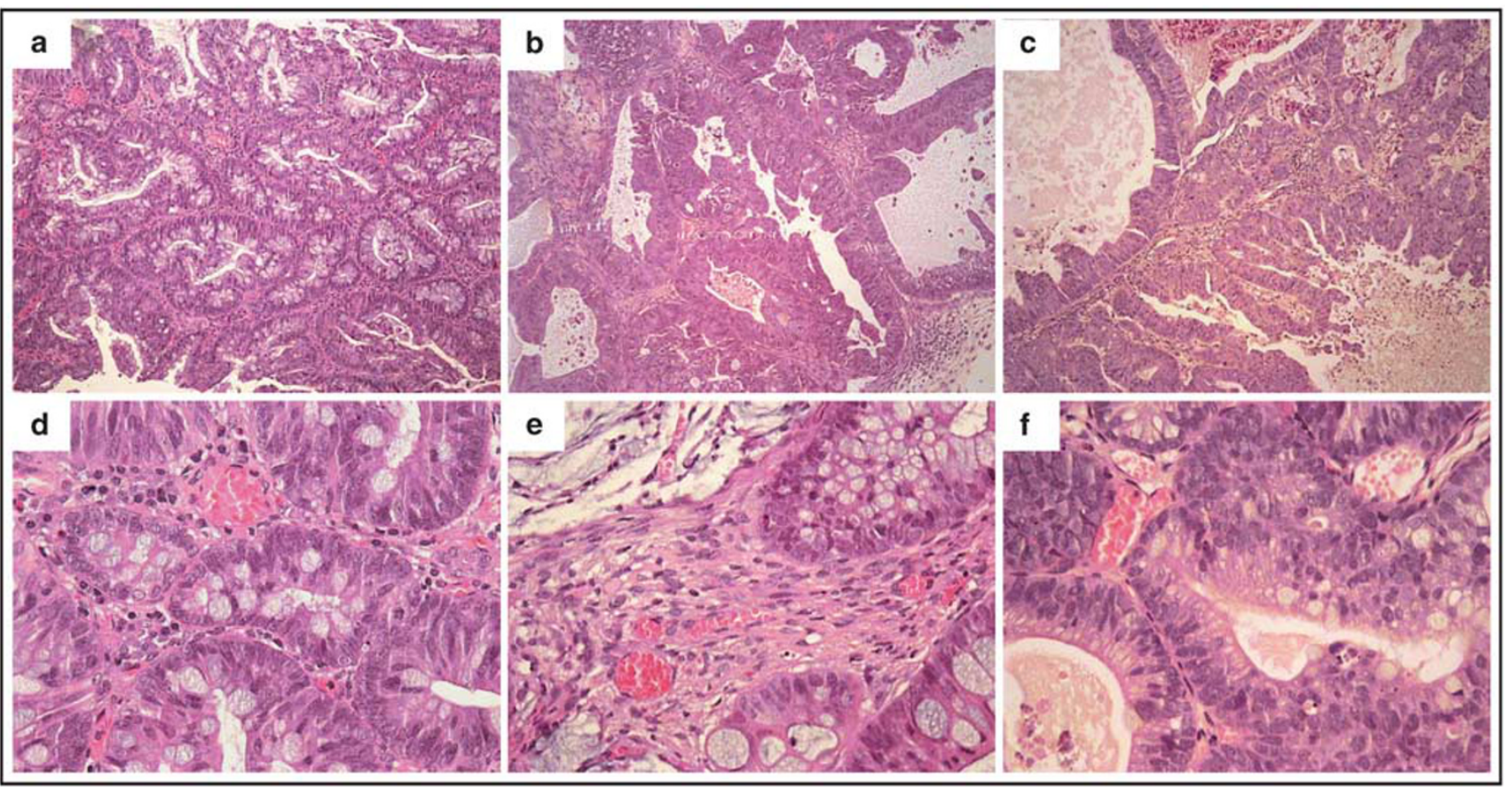

Figure 1 Histological appearance of an initial CRC tissue specimen (a) and two xenografts derived from the same specimen and resected 10 (b) and 30 (c) days after implantation. H\&E; original magnification, $\times 100$. Presence of erythrocytes in the lumen of blood vessels in an initial tissue specimen (d) and xenografts harvested 10 (e) and 30 (f) days postimplantation. H\&E; original magnification, $\times 400$. 
sues. Both mAbs labeled ECs in human specimens, but did not label ECs in mouse tissue (data not shown).

Xenograft establishment is presumed to depend on reperfusion via anastomosis with host vessels. Therefore, the origin of the vasculature in the xenografts was analyzed using antimouse CD31 and CD34 mAbs. The species specificity of a rat $\mathrm{mAb}$ antimouse $\mathrm{CD} 34$ was demonstrated using human initial CRC (Supplementary Figure 1c) and mouse control tissues (data not shown). In contrast, the antimouse CD31 antibody (clone MEC13.3) failed to label mouse ECs in mouse tissue, in spite of previous reports of using this clone in formalin-fixed paraffin sections. Consequently, the species of origin of the vasculature in the CRC xenografts was determined by IHC analysis of serial sections with antihuman and antimouse CD34 mAbs (Figure 2). The number of vessels positive for each of the species-specific CD34 antibodies was counted in five fields per section and averaged. Immunostaining of preimplantation CRC tisssue sections is shown in Figure 2a and b. By day 10, both markers failed to stain all of the vessels in the tissue specimens and generally stained approximately equal number of vessels, with areas where human vessels predominated (Figure $2 \mathrm{c}$ and $\mathrm{d}$ ) and areas where the vessels were exclusively murine (Figure $2 \mathrm{e}$ and f). By day 20, vessels of murine origin predominated over human ones (data not shown) and by day 30, no staining could be detected with the antihuman CD34 antibody (Figure $2 \mathrm{~g}$ ), although all vessels were positive for antimouse CD34 (Figure 2h). Therefore, vessel density was kept nearly constant thanks to the recruitment of murine ECs that progressively colonized the xenograft. Quantification of temporal changes in vessel density and species reactivity is shown in Figure 2i. Absence of human endothelium at day 30 was corroborated using the antihuman $\mathrm{CD} 31 \mathrm{mAb}$ to rule out a selective downregulation of human CD34 in ECs (data not shown).

\section{Double Immunolabeling of Human Endothelial Cell Apoptosis}

To evaluate if human blood vessels undergo apoptosis soon after transplantation, we performed a newly developed double immunolabeling technique with anti-CD34 and antiactivated caspase-3. ${ }^{19}$ Classically, visualization of apoptosis of ECs was performed using the terminal transferase-mediated dUTP nick end-labeling (TUNEL) method. However, the low sensitivity makes the TUNEL staining method unreliable to identify apoptotic cells. ${ }^{20}$

In tissue sections from day 10 after xenograft, we could identify human CD34-negative and anti-activated caspase3-positive staining of scarce tumor cells dispersed throughout the specimen. Vessels lined with antihuman CD34-positive cells could also be clearly discerned. However, we were not able to demonstrate double immunostaining of human ECs with both anti-CD34 and anti-activated caspase-3 (data not shown).

\section{Colocalization of Murine Endothelial Cells and Human Basement Membranes}

Vascular replacement has been proposed as a critical mechanism of human skin graft revascularization in mouse models, with involution of donor graft ECs coincident with ingrowth of ECs from the host. ${ }^{22,23}$ What is more, migration of mouse ECs into the graft was shown to take place over the basement membrane (BM) of previous human vessels. ${ }^{22,23}$ In line with these findings, we wanted to know if this event could also be demonstrated in our model of CRC xenograft. To this aim, sections were double-labeled with mAbs antimouse CD34 and antihuman laminin (clone LAM-89). According to the manufacturer, this antibody shows no crossreactivity with murine laminin. Interestingly, at day 10 after implantation, we could detect vessels lined with murine ECs and surrounded by a BM containing human laminin (Figure 2j). However, at day 30, the presence of human laminin could not be demonstrated, suggesting the production of a new BM by mouse ECs (data not shown).

\section{Establishment of Human Renal Cell Carcinoma Primary Xenografts and Species-Specific Vascular Labeling}

To rule out that the differences observed between the fate of human vasculature in our CRC model and those previously reported were because of methodological aspects, we established RCC primary xenografts in immunodeficient mice using the same protocol. The majority of vessels in RCC xenografts, as well as prostate xenografts, have been shown to be lined with human ECs 30 days after implantation. ${ }^{18}$ Mice were killed at day 35, and xenografts were resected. Macroscopically, engrafted xenografts were surrounded by visible host vessels penetrating the tumor mass. The proportion of surgical specimens that yielded at least one viable xenograft was $100 \%$, with per fragment take rates of $61 \%(n=18)$.

Staining with H\&E of RCC xenograft sections revealed a typical clear cell histology, with nests of carcinoma cells separated by a network of small blood vessels. Subsequently, the origin of the vasculature in RCC xenografts was analyzed by IHQ, using species-specific anti-CD34 mAbs. In marked contrast with CRC xenografts, vasculature within cancer cell nests in RCC xenografts was mostly of human origin (Figure 3a), with some interspersed host-derived cells (Figure 3b). ECs in the thin connective tissue layer at the periphery, presumably of host origin, were stained with antimouse CD34. Interestingly, in some specimens an angiomatoid-like reaction was observed in the tumor-associated stroma. The numerous blood vessels in these areas were exclusively human in nature (Figure 3c), with murine vessels clearly limited to the peripheral strip of connective tissue (Figure 3d). Quantification of vessel density and species reactivity yielded a mean number of 145 human microvessels per field $v s 28.75$ of murine origin, being most of the latter identified by a single CD34-positive cell and/or circumscribed to the periphery. 



Figure 2 Immunohistochemical analysis of preimplantation (day 0) CRC tissue sections and xenografts resected 10 and 30 days after implantation using antihuman CD34 (a, c, e and $\mathbf{g}$ ) and antimouse CD34 (b, d, $\mathbf{f}$ and $\mathbf{h})$. Original magnification, $\times 400$. (i) Temporal changes in vessel density (mean \pm s.d. of CD34-positive vessels in five high-power fields per section). Quantification of vasculature in CRC initial specimens and xenografts harvested 10, 20 or 30 days after implantation. (j) Double immunostaining (detail) of mouse CD34 (DAB) and human laminin (permanent red) of xenograft sections 10 days postimplantation. Solid arrows indicate murine ECs (reddish brown), and arrowheads the laminin-rich basement membrane (pink). Original magnification, $\times 1000$, immersion oil.

\section{DISCUSSION}

This study established that an angiogenic response was not elicited from preexisting human vessels after xenograft establishment of CRC specimens. In addition, human vessels did not persist in the xenograft and were rapidly substituted by vessels lined with murine ECs. The areas of necrosis observed at day 30 postimplantation suggest that murine angiogenesis is insufficient to maintain the entire cellular mass during xenograft growth. These results are in marked contrast to previous data obtained from RCC and prostate carcinoma primary xenografts, where human vessels (identified with the antihuman $\mathrm{CD} 31 \mathrm{mAb}$ used in this work, clone JC/70A) persisted for 1 month (at least $80 \%$ of the vasculature) after implantation in athymic nude mice. ${ }^{18}$ In another study with human primary breast cancer transplants in NOD/Scid mice, most of the explanted xenografts exhibited blood vessels reactive with the same antihuman CD31 antibody 11 weeks posttransplantation. ${ }^{24}$ Similarly, human uveal malignant melanoma primary xenografts in nude mice, harvested when the tumors reached approximately $10 \mathrm{~mm}$ in diameter, also contained in the vascular septa anti-CD31 (clone JC/70A) and anti-CD34 (clone My10)-positive cells. ${ }^{25}$ However, in the two latter studies, the presence of murine ECs was not analyzed, so colonization of xenografts by murine vessels cannot be ruled out. It is noteworthy that the genetic background of the mice used in this study is the same in two out of the three works mentioned. 
Anti-human CD34

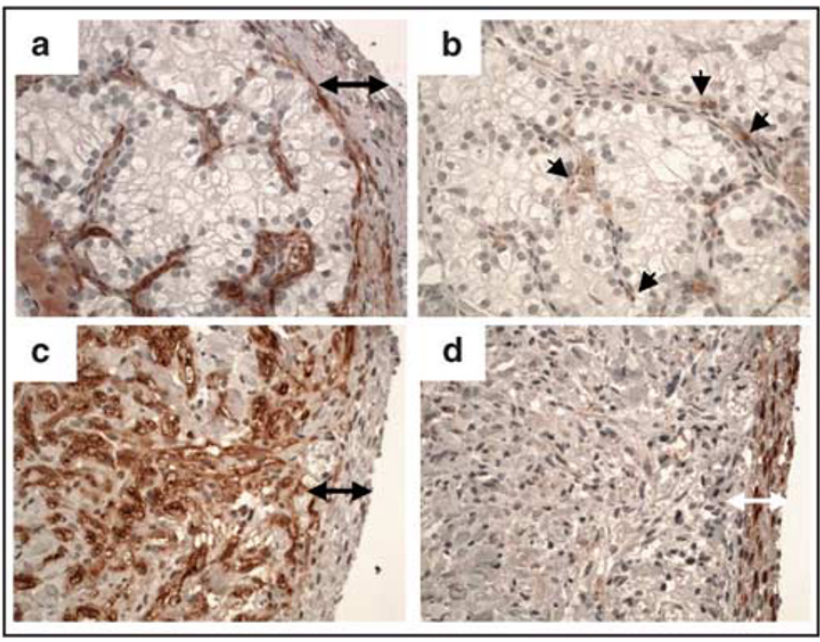

Figure 3 Photomicrographs of representative fields $(\times 400)$ showing human ( $\mathbf{a}$ and $\mathbf{c}$ ) and murine (b and $\mathbf{d}$ ) CD34 staining of microvessels in RCC xenografts 35 days postimplantation. (a and b) RCC xenograft area with typical clear cell histology. (c and d) Area of angiomatoid-like reaction in nearby stroma. Arrowheads indicate ECs stained with antimouse CD34. Double-headed arrows indicate the peripheral strip of connective tissue presumably of host origin.

The apparent discrepancies between these earlier works and ours could be attributed to methodological differences, given that although exquisite care was taken to minimize ischemic time and preserve specimen architecture, xenograft implantation procedures were not identical. To achieve minimal invasiveness, we used a small trocar device that required no previous surgical incision, but could have led to more extensive dissociation of cells within the tumor specimen. To discard this possibility, RCC xenografts were established using the same protocol, and mice were killed after 35 days. In all specimens analyzed by IHQ, human ECs were detectable and constituted the main component of blood vessels, with a few murine cells contributing to tumor vasculature and being mainly confined to the periphery of the xenografts.

Taken together, these data strongly suggest that the fate of endothelium in primary xenografts of different origin relies on intrinsic tumor-specific microenvironmental cues. It is known that the intensity of the angiogenic response varies considerably within different zones of a single tumor ${ }^{26}$ and between different types of tumors. ${ }^{27}$ In fact, CRC and CRC liver metastases yielded the lowest microvessel density in a panel of human tumors that included lung, kidney, gastric, breast, prostate and pancreatic carcinomas. ${ }^{20}$

Histologically, human carcinomas are composed of both parenchyma and stroma. Tumor-associated stromal cells (ECs, fibroblasts, smooth muscle cells and inflammatory cells, all embedded in abundant extracellular matrix) interact with each other and the cancer cells in a complex fashion and are thought to generate a favorable microenvironment for cancer cell survival. ${ }^{28}$ In this respect, EC survival may depend on the production of angiogenic factors, such as VEGF-A, by stromal cells in turn activated by growth factors released by cancer cells. ${ }^{29,30}$ Tumor stroma, as a provider of mural cells and their corresponding growth factors, is also responsible for vessel maturation. ${ }^{31}$ It could be argued that vascular heterogeneity in tumors could arise from tumor stroma heterogeneity. Therefore, we could speculate about the existence of tumor-associated stromal cells with a high/low angiogenic profile that would determine the differences in microvessel density observed, for instance, between CRC and RCC. In the case of CRC, a low angiogenic profile could explain why 'passenger' ECs in xenografts did not survive after a radical microenvironmental change. Subsequent tumor hypoxia would explain murine EC recruitment.

EC apoptosis has been proved to play a role in remodeling during tumor angiogenesis. ${ }^{20}$ To test if human EC substitution could be attributed to apoptotic death, we used double immunolabeling with antihuman CD34 and anti-activated caspase-3. At day 10, human ECs were unequivocally identified and some apoptotic tumor cells were detected, but we could not demonstrate apoptotic human ECs. These data suggest that apoptosis is a very early event after implantation or, alternatively, it is not responsible for the loss of human ECs. The molecular mechanisms underlying the disappearance of human ECs in this primary CRC xenograft model deserve further study.

Mouse ECs not only colonize human skin grafts but also migrate following the $\mathrm{BM}$ tracts of preexisting human vessels. $^{22,23}$ The appearance of these 'BM ghosts' has also been documented in other contexts, such as regression of tumor vessels after inhibition of VEGF signaling, and they have been proposed as potential scaffolds for vessel regrowth. ${ }^{32}$ We wanted to know if this event could also take place in the CRC xenograft model. To address this question, sections were double stained with $\mathrm{mAbs}$ recognizing mouse CD34 and human laminin. Laminin is one of the main components of $\mathrm{BM}$, and has a key role in vessel formation and stability. ${ }^{33}$ We found vessels composed of mouse CD34-positive ECs and enveloped by a BM-containing human laminin in day 10 sections. By day 30, however, human laminin could not be detected, implying the formation of an entirely murine vascular BM. These data suggest that initial colonization of CRC xenografts by murine ECs could take advantage of preexisting BM. It could be interesting to evaluate if this process plays also a role in the spontaneous remodeling of vasculature during tumor growth. Functionally, active sites within vascular BM might therefore represent an interesting therapeutic target to suppress vascular regrowth. ${ }^{33}$

In conclusion, our results suggest that special care should be taken in the analysis of the nature of EC in primary xenografts, when proposed as in vivo models of human tumor vasculature, given the contradictory results observed with different histological types of carcinoma. This discrepancy could be attrib- 
uted to differences, despite their common epithelial origin, in the angiogenic profile of the tumors.

Supplementary Information accompanies the paper on the Laboratory Investigation website (http://www.laboratoryinvestigation.org)

\section{ACKNOWLEDGEMENTS}

We thank MJ Acuña and L Kilany for expert technical assistance. LS is investigator from the Ramón y Cajal Program (Ministerio de Ciencia e Innovación, cofinanced by the European Social Fund). This study was partially supported by grants from Fondo de Investigación Sanitaria (PI061621) to LS, and from Ministerio de de Ciencia e Innovación (BIO200504794), Comunidad Autónoma de Madrid (S-BIO-0236-2006) and Fundación Mutua Madrileña to LA-V.

1. Hanahan D, Folkman J. Patterns and emerging mechanisms of the angiogenic switch during tumorigenesis. Cell 1996;86:353-364.

2. Naumov GN, Bender $E$, Zurakowski $D$, et al. A model of human tumor dormancy: an angiogenic switch from the nonangiogenic phenotype. J Natl Cancer Inst 2006;98:316-325.

3. Ferrara N, Kerbel RS. Angiogenesis as a therapeutic target. Nature 2005;438:967-974.

4. Sanz $L$, Alvarez-Vallina $L$. The extracellular matrix: a new turn-of-thescrew for anti-angiogenic strategies. Trends Mol Med 2003;9:256-262.

5. Sanz L, Pascual M, Muñoz A, et al. Development of a computer-assisted high-throughput screening platform for anti-angiogenic testing. Microvasc Res 2002;63:335-339.

6. Norrby K. In vivo models of angiogenesis. J Cell Mol Med 2006;10: 588-612.

7. Kelland LR. Of mice and men: values and liabilities of the athymic nude mouse model in anticancer drug development. Eur J Cancer 2004;40:827-836.

8. Nör JE, Peters $M C$, Christensen JB, et al. Engineering and characterization of functional human microvessels in immunodeficient mice. Lab Invest 2001;81:453-463.

9. Skovseth DK, Yamanaka T, Brandtzaeg P, et al. Vascular morphogenesis and differentiation after adoptive transfer of human endothelial cells to immunodeficient mice. Am J Pathol 2002;160:1629-1637.

10. Enis DR, Shepherd BR, Wang $Y$, et al. Induction, differentiation, and remodeling of blood vessels after transplantation of $\mathrm{BCl}$-2-transduced endothelial cells. Proc Natl Acad Sci USA 2005;102:425-430.

11. Koike $N$, Fukumura $D$, Gralla $O$, et al. Tissue engineering: creation of long-lasting blood vessels. Nature 2004;428:138-139.

12. Dong Z, Neiva KG, Jin T, et al. Quantification of human angiogenesis in immunodeficient mice using a photon counting-based method. Biotechniques 2007;43:73-77.

13. Sanz $\mathrm{L}$, Santos-Valle $\mathrm{P}$, Alonso-Camino $\mathrm{V}$, et al. Long-term in vivo imaging of human angiogenesis: critical role of bone marrow-derived mesenchymal stem cells for the generation of durable blood vessels. Microvasc Res 2008;75:308-314.

14. Kumar $\mathrm{P}$, Ning $\mathrm{Y}$, Polverini PJ. Endothelial cells expressing BCl-2 promotes tumor metastasis by enhancing tumor angiogenesis, blood vessel leakiness and tumor invasion. Lab Invest 2008:88:740-749.

15. Morton CL, Houghton PJ. Establishment of human tumor xenografts in immunodeficient mice. Nat Protoc 2007:2:247-250.
16. Visonneau S, Cesano A, Torosian MH, et al. Growth characteristics and metastatic properties of human breast cancer xenografts in immunodeficient mice. Am J Pathol 1998;152:1299-1311.

17. Vassal G, Terrier-Lacombe MJ, Lellouch-Tubiana A, et al. Tumorigenicity of cerebellar primitive neuro-ectodermal tumors in athymic mice correlates with poor prognosis in children. Int J Cancer 1996;69: 146-151.

18. Gray DR, Huss WJ, Yau JM, et al. Short-term human prostate primary xenografts: an in vivo model of human prostate cancer vasculature and angiogenesis. Cancer Res 2004;64:1712-1721.

19. Heijstek MW, Kranenburg O, Borel Rinkes IH. Mouse models of colorectal cancer and liver metastases. Dig Surg 2005;22:16-25.

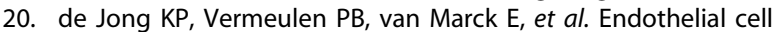
apoptosis in the context of quantification of angiogenesis in solid human adenocarcinomas: a novel double immunolabelling technique to identify endothelial cell apoptosis. Eur J Cancer 2006;42:97-100.

21. Vermeulen PB, Gasparini G, Fox SB, et al. Second international consensus on the methodology and criteria of evaluation of angiogenesis quantification in solid human tumours. Eur J Cancer 2002;38:1564-1579.

22. Demarchez $M$, Hartmann DJ, Prunieras $M$. An immunohistological study of the revascularization process in human skin transplanted onto the nude mouse. Transplantation 1987;43:896-903.

23. Capla JM, Ceradini DJ, Tepper OM, et al. Skin graft vascularization involves precisely regulated regression and replacement of endothelial cells through both angiogenesis and vasculogenesis. Plast Reconstr Surg 2006;117:836-844.

24. Beckhove P, Schutz F, Diel IJ, et al. Efficient engraftment of human primary breast cancer transplants in nonconditioned NOD/Scid mice. Int J Cancer 2003:105:444-453.

25. Heegaard S, Spang-Thomsen M, Prause JU. Establishment and characterization of human uveal malignant melanoma xenografts in nude mice. Melanoma Res 2003;13:247-251.

26. Achilles EG, Fernandez A, Allred EN, et al. Heterogeneity of angiogenic activity in a human liposarcoma: a proposed mechanism for 'no take' of human tumors in mice. J Natl Cancer Inst 2001;93: 1075-1081.

27. Eberhard A, Kahlert S, Goede V, et al. Heterogeneity of angiogenesis and blood vessel maturation in human tumors: implications for antiangiogenic tumor therapies. Cancer Res 2000;60:1388-1393.

28. Liotta LA, Kohn EC. The microenvironment of the tumour-host interface. Nature 2001;411:375-379.

29. Kitadai Y, Sasaki T, Kuwai T, et al. Targeting the expression of plateletderived growth factor receptor by reactive stroma inhibits growth and metastasis of human colon carcinoma. Am J Pathol 2006;169:2054-2065.

30. Duda DG, Fukumura D, Munn LL, et al. Differential transplantability of tumor-associated stromal cells. Cancer Res 2004;64:5920-5924.

31. Jain RK. Molecular regulation of vessel maturation. Nat Med 2003;9:685-693.

32. Inai $\mathrm{T}$, Mancuso $\mathrm{M}$, Hashizume $\mathrm{H}$, et al. Inhibition of vascular endothelial growth factor (VEGF) signaling in cancer causes loss of endothelial fenestrations, regression of tumor vessels, and appearance of basement membrane ghosts. Am J Pathol 2004;165:35-52.

33. Sanz L, García-Bermejo L, Blanco FJ, et al. A novel cell binding site in the coiled-coil domain of laminin involved in capillary morphogenesis. EMBO J 2003:22:1508-1517. 